\title{
ORTHODONTIC PROBLEMS IN PATIENTS WITH HYPODONTIA AND TAURODONTISM OF PERMANENT MOLARS
}

\author{
Miroslava Yordanova ${ }^{1}$, Svetlana Yordanova ${ }^{1}$, G. Tomov ${ }^{2}$ \\ 1) Department of Orthodontics, \\ 2) Department of Dental Surgery and Endodontics, \\ Faculty of Dentistry, Medical University, Plovdiv
}

\begin{abstract}
Taurodontism is a morphological abnormality of the tooth shape with the following characteristic features: vertically enlarged pulp chamber, apical displacement of the bifurcation and trifurcation, shortened roots and no constriction at the level of the cementoenamel junction. This anomaly is still neglected by orthodontists when it comes to making treatment plans in spite of the reports in the literature of a close association of this condition with hypodontia and oligodontia.

Aim: The article discusses the orthodontic problems arising in treating patients with hypodontia and taurodontism and suggests approaches to reducing the risk of complications during orthodontic tooth movement.

Discussion: Failure to identify taurodont teeth in hypodontic patients can cause serious complications in their orthodontic management because of the greater susceptibility of these types of teeth to root resorption in orthodontic tooth movement. Besides, these types of teeth are less anchorage.

Conclusion: The high incidence of taurodontism in hypodontic patients presents a clinical challenge for general dental practitioners and orthodonists alike. Furthermore, successful management of hypodontia rests on the orthodontist's ability to identify taurodont teeth and correctly include them in treatment plan.
\end{abstract}

Key words: taurodontism, taurodont teeth, hypodontia, root resorption

\section{INTRODUCTION}

The term taurodontism was first introduced by Arthur Keith in 1913 (1) when this dental anomaly was observed in the skulls of the Neanderthal man.

Taurodontism is a morphological abnormality of the tooth shape. The pulp chamber of the taurodont tooth is enlarged vertically, the bifurcation and trifurcation are displaced apically, the roots are shortened and there is no constriction at the level of the cementoenamel junction. The incidence of this anomaly differs from population to population. Permanent dentition is most commonly affected, although taurodontism can occur in primary dentition too. The most frequently affected teeth are the molars though premolars are also known to be affected. Taurodontism can be isolated anomaly, but it can be concomitant with several severe developmental syndromes. Taurodontism is still neglected by orthodontists when it comes to making treatment plans in spite of reports in the literature of a close association of this condition with hypodontia and oligodontia $(2,3)$.

\section{AIM}

The article discusses the orthodontic problems arising in treating patients with hypodontia and taurodontism and suggests approaches to reducing the risk of having complications during orthodontic tooth movement.

\section{MATERIAL AND METHODS.}

In the clinical cases herewith reported of patients with hypodontia the diagnosis of taurodontism was made by orthopantomography (OPG); we used the method of Shifman and Chanannel (4) for teeth with complete development of at least half of the root length and the method of Blumberg (5) for teeth with incomplete root development. The pulp chamber height and the root canal length were measured with a ruler to the nearest $0.5 \mathrm{~mm}$ and used to determine the taurodont index $\mathrm{TI}=\mathrm{d} 1 \backslash \mathrm{d} 2 \times 100$ (Fig. 1). Taurodontism was diagnosed at TI greater than 20. In teeth with incompletely developed roots we used the measure $\mathrm{d} 3$ (Fig. 2 ), which is indicative of taurodontism if greater than 3.5 $\mathrm{mm}$. The severity of taurodontism (hypo-, meso, or hypertaurodontism) was also determined by the method of Shifman and Chanannel (4). 


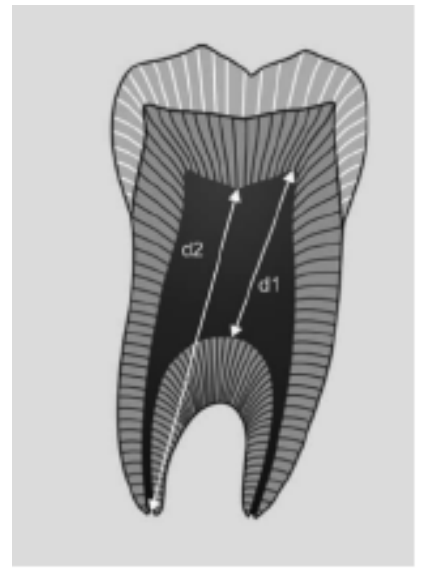

Fig. 1.

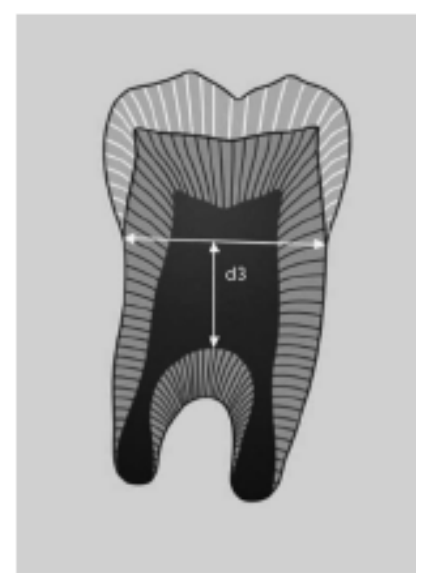

Fig. 2.

Fig. 1. Taurodont index of permanent teeth.

Fig. 2. Taurodontism of permanent teeth with incomplete root development.

\section{Case report 1}

P. B., a 13-year-old girl, was referred for orthodontic treatment by her dental practitioner with the diagnosis of hypodontia of teeth 12, 35, and 45 (Fig. 3).

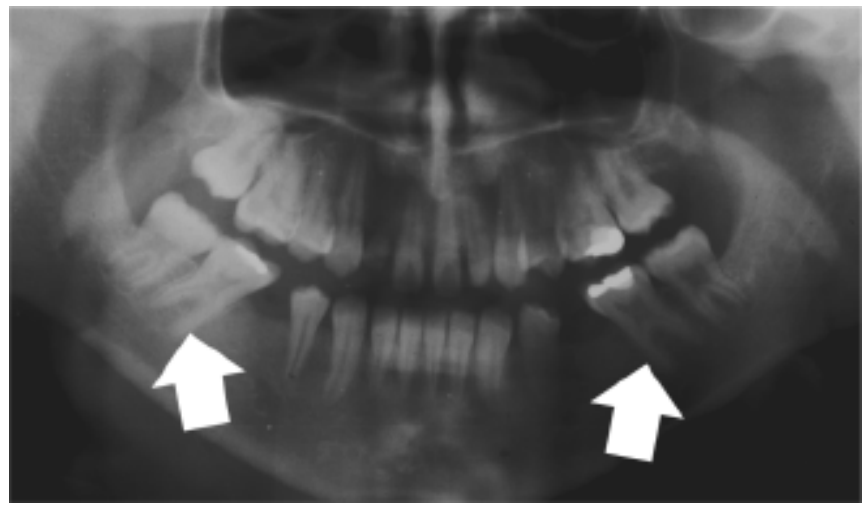

Fig. 3a.

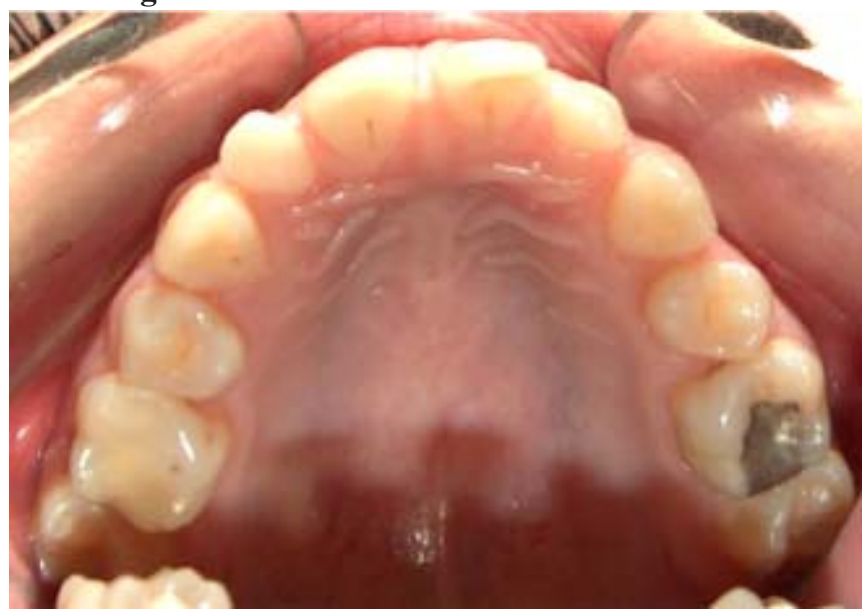

Fig. 3a.

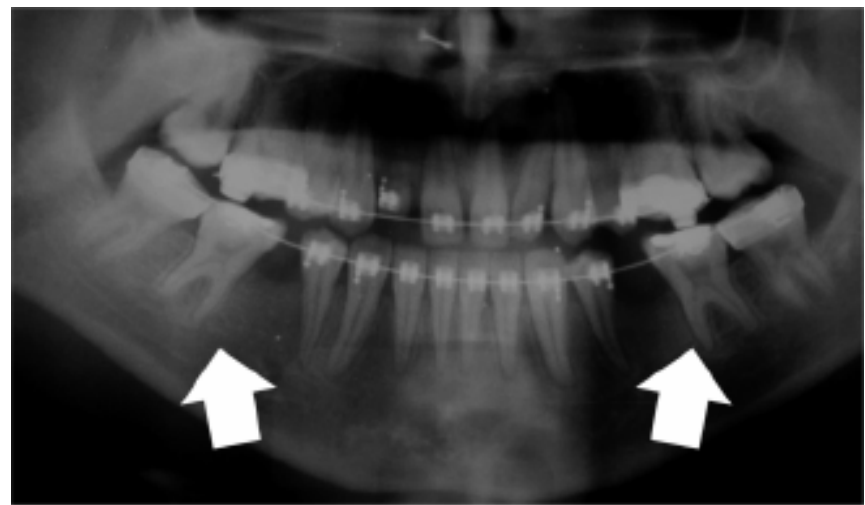

Fig. 3b.

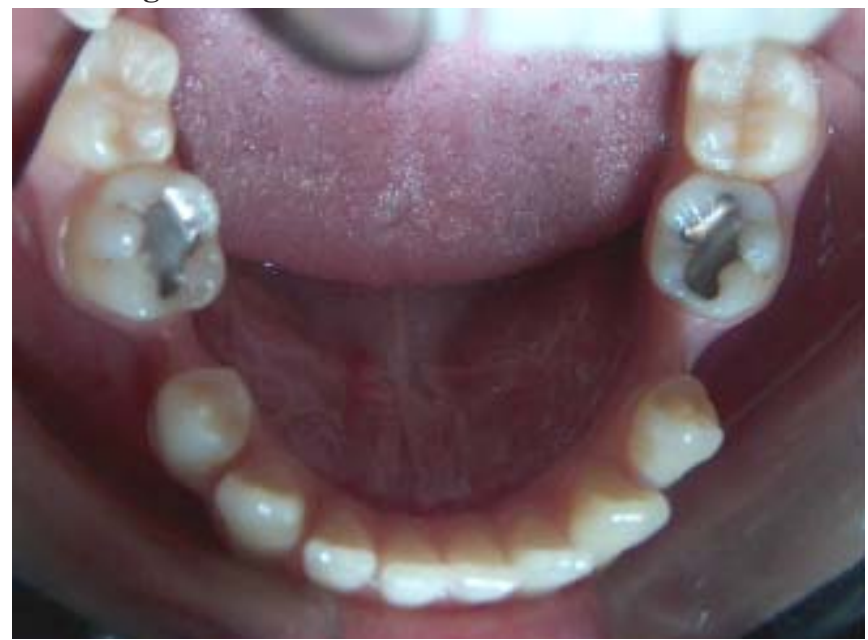

Fig. 3b.

Figure 3. Patient P B., 13 years old.

The findings after a battery of comprehensive clinical, photometric and teleroentgenographic studies were: overretained deciduous tooth 52 , hypodontia of teeth 12,35 , and 45 ; improperly aligned upper and lower permanent teeth with gaps and diastema in the upper jaw; VL-reduction of alveolar bone in the edentulous premolar areas; Angle class I dental relationships, skeletal class III, edge to edge bite in teeth 22,23 , and 33 , and 43 , hyperdivergent type of growth. Unaesthetic facial features with a positive lip step, protrusive chin and flattened labiomental fold.

The radiographic examination with OPG revealed hypotaurodontism of teeth 36 and 46.

The treatment plan included a fixed vestibular multibracket appliance with restoration of the proper shape and size of teeth 11,21 , and 22, levelling and alignment of dental arches, and preserving class I occlusion in the lateral segments. After taking into account all diagnostic parameters and discussing the treatment with the patient, to restore the missing tooth 12 we chose to perform extraction of tooth 52 , make some space and place an orthopaedic implant. Because teeth 36 and 46 were taurodontic, the mandibular hypodontia was treated by preserving the edentulous spaces for future 
prosthetic restoration. Orthodontic medialization of the first molars to close the gaps was rejected in the plan as this procedure carries a risk of root resorption .

\section{Case report 2}

A 9-year-old girl R.B. was referred for orthodontic treatment by her dental practitioner with a diagnose of hypodontia of all permanent second premolars. Clinical and paraclinical studies revealed a mixed dentition with gaps and diastema, Angle class I dental relationships, class I skeletal relations, deep bite, normodivergent facial pattern and pleasing facial features. The examination with OPG revealed also taurodontism of teeth 37 and 47.

The patient's hypodontia was managed by aligning the dental arches with the existing permanent teeth. The vertical problem will be dealt with by levelling the dental arches and intrusion of the upper frontal teeth. The treatment started with early extraction of the primary molars (9 years old). The ensuing dental drift of teeth $36,46,37$, and 47 reduced greatly the edentulous spaces in the mandible. Thus we achieved save medialization of the taurodont molars until gaps were fully closed. In R. B. the residual small gaps will be closed orthodontically with minimal force under constant radiographic control using a multibracket appliance (Fig.4).

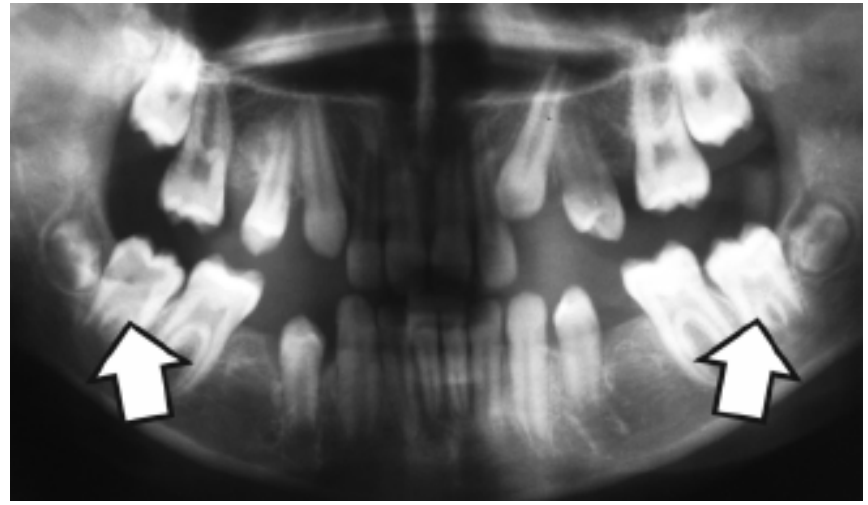

Fig. 4a.

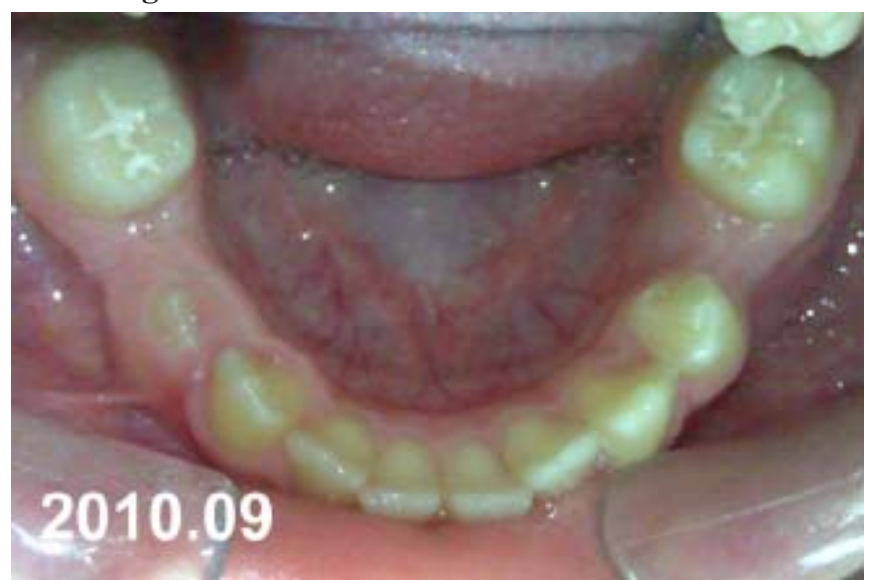

Fig. 4b.

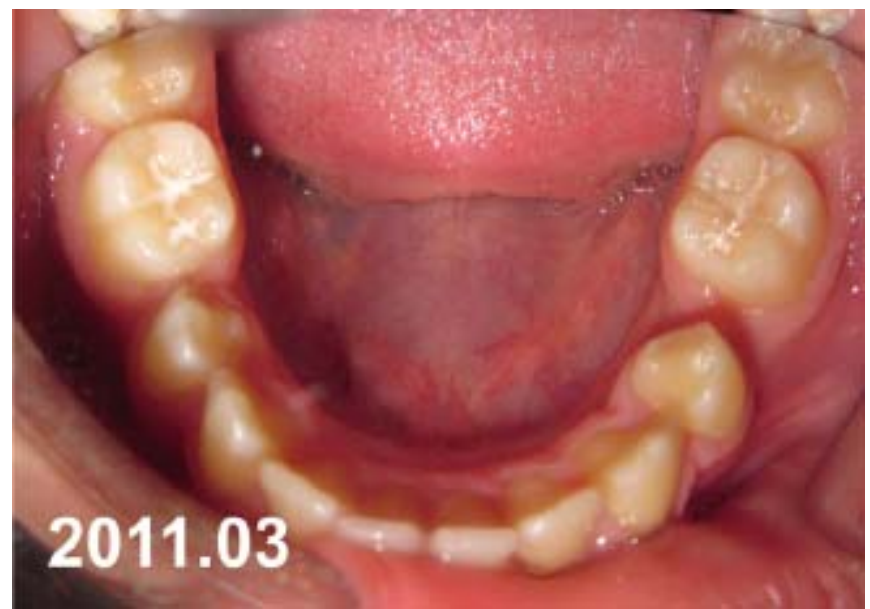

Fig. 4c.

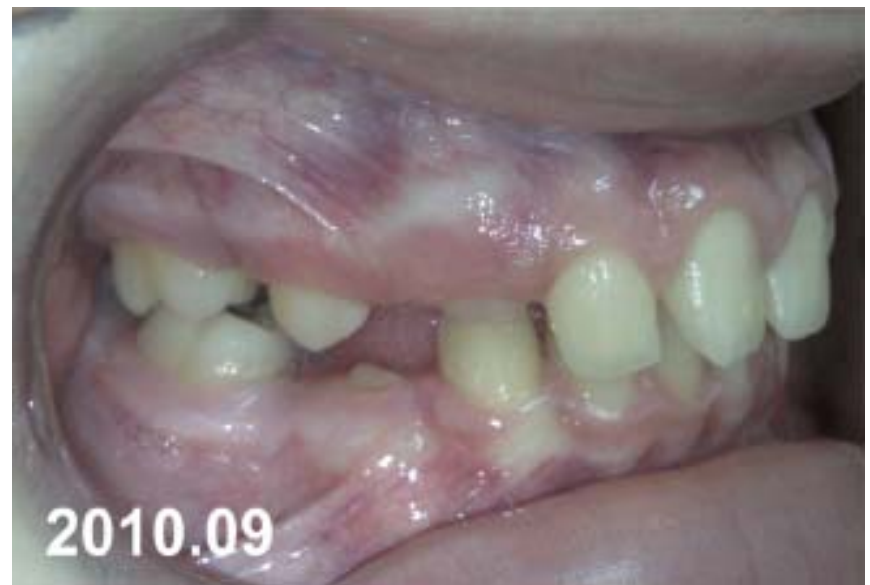

Fig. 4d.

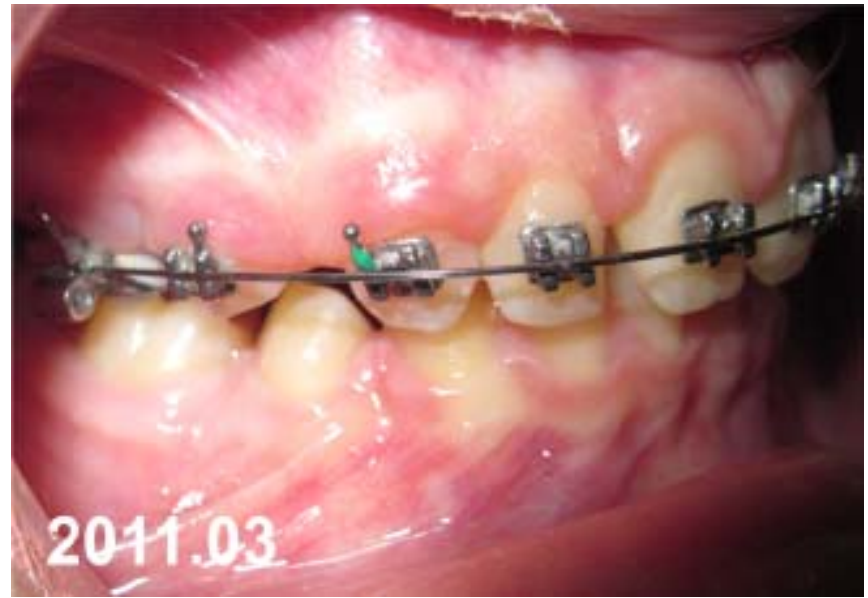

Fig. 4e. 


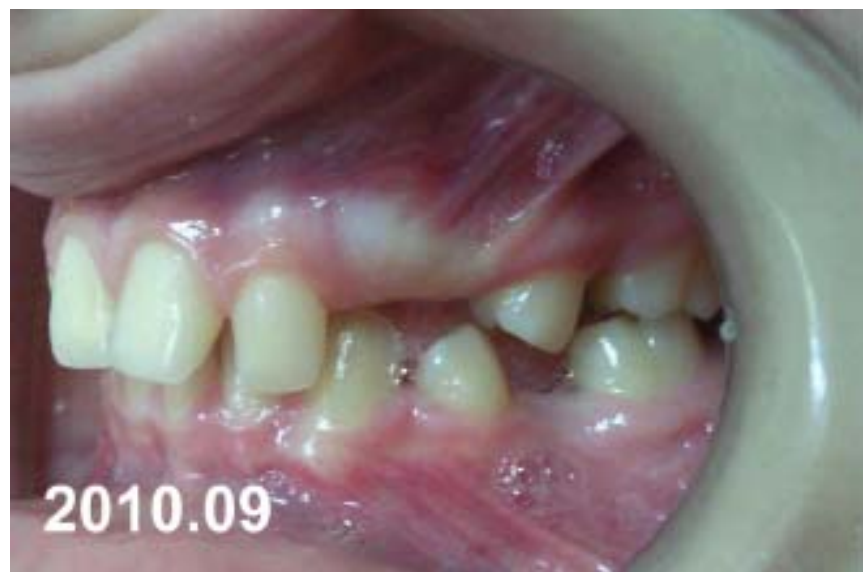

Fig. 4 f.

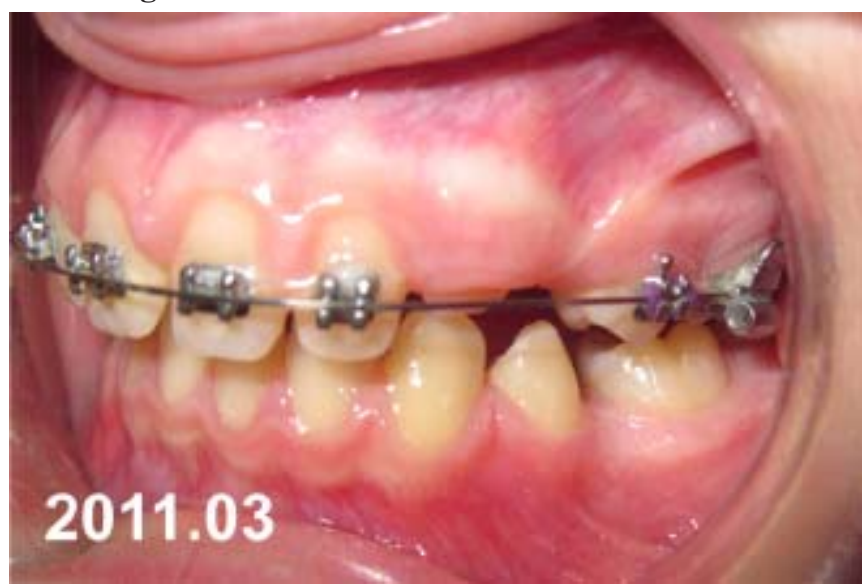

Fig. 4g.

Figure 4. Patient R. B., 10 years old

\section{DISCUSSION}

Although this anomaly is considered to be an atavistic feature by some researchers taurodontism has been frequently reported in present day man with varying incidence rate ranging from $0.25 \%$ to $18 \%$ (1). There are reports in the relevant literature of considerable increase the taurodontism incidence in patients with hypodontia and oligodontia compared with the frequency of the nonsyndrome isolated form. It is $29 \%$ in Dutch patients (6) and $21.2 \%$ in Brazilian patients (7). Seow and Lai studied a sample of 66 hypodontia patients and found the prevalence of the condition to be $34.8 \%$ (3). Stenvik and Zachrisson found in their study sample almost one third of the mandibular first molars to be taurodont teeth (8). The results of our previous study (37.5\%) (9) are consistent with those researchers studying the incidence of taurodontism in Caucasian patients with hypo- and oligodontia.

Because of the greater incidence rate of taurodontism in Bulgarians with hypodontia and oligodontia such patients must merit circumspect diagnosing process. Failure to recognize typical morphological characteristics of taurodont teeth and to register them in the patient's status card can seriously undermine the proper choice of treatment modality.

Using conventional orthodontic techniques for medialization to close the edentulous hypodontic sections is not the best option for patients with retrusive or flat facial profile. Besides the aesthetic considerations, however, another contraindication of orthodontic movement of premolars or molars in the cases with hypodontia is that they should exhibit signs of taurodontism as in case 1. The shorter and thinner roots of taurodont teeth are considerably more susceptible to resorption when orthodontic forces are applied for their movement (10).

The recommended approach in such cases is to limit the orthodontic teeth movement to only preparation procedures to facilitate any future prosthetic rehabilitation with fixed constructions. What is mainly needed is to upright the medially inclined molars or reposition them (medially or distally) over a small distance, should the teeth be used as abutments in future fixed constructions.

If in managing hypodontia you have chosen to close the gaps by conventional orthopaedic constructions supported by taurodont teeth (be that a partial or fixed prosthetic construction) you should also take into account the teeth's long-term stability, the reason being that these teeth are poor support when used as abutments. Their smaller root surface makes them become unstable when additional force is applied (11).

When the treatment plan involves closing of spaces using the available teeth, for patients with hypodontia and taurodontism of molars, as in case 2, it is advisable that you should choose spontaneously closing gaps. This is possible with early extraction (8-9 years olds) of primary molar teeth. Permanent molars are medialized naturally and the residual gaps remain within 1 to $2 \mathrm{~mm}$. Thus the shorter distance significantly reduces the risk of root resorption in orthodontic movement of molars until all gaps are closed.

These cases are examples of how taurodontism influences the choice of treatment modality. It is of particular importance for adolescent patients with hypodontia and oligodontia to have their taurodont teeth preserved having in mind their already reduced number of teeth and the future bone growth. Therefore, the orthodontist should be thoroughly familiar with this dental anomaly so that it can be detected in time.

\section{CONCLUSION}

The high incidence of taurodontism in hypodontic patients presents a clinical challenge for general dental practitioners and orthodonists alike. To successfully manage hypodontia the orthodontist should be able to identify taurodont teeth and correctly include them in the treatment plan. 


\section{REFERENCES:}

1. Manjunatha BS, Kovvuru S. Taurodontism - a review on its etiology, prevalence and clinical considerations. J Clin exp Dent 2010; 2(4): 187-90.

2. Schalk-van-der Weide Y, Steen WH, Bosman F. Taurodontism and length of teeth in patients with oligodontia. J Oral Rehabil 1993 Jjul;20(4):401-412. [PubMed]

3. Seow WK, Lai PY. Association of taurodontism with hypodontia: a controlled study. Pediatr Dent 1989 Sep;11(3):214-19. [PubMed]

4. Schifman A, Chanannel I. Prevalence of Taurodontism in radiographic dental examination of 1200 young adult Israeli patients. Community Dent Oral Epidemiol. 1978 Jul;6(4):200-203. [PubMed]
5. Blumberg JE, Hylander WL, Goepp RA. Taurodontism: a biometric study. Am J Phys Antropol. 1971 Mar;34(2):243-55. [PubMed]

6. Schalk-van-der Weide Y. Oligodontia. A clinical, radiographic and genetic evaluation. Thesis. Utrecht, University of Utrecht 1992.

7. Gomes RP, da Fonseca JA, Paula LM, Faber J, Acevedo AC. Prevalence of hypodontia in orthodontic patients in Brazil. Eur J Orthod 2010 June;32(3):302-6. DOI: 10.1093/ejo/cjp107 CrossRef] [PubMed]

8. Stenvik A, Zachrisson BU, Svatun B. Taurodontism and concomitant hypodontia in siblings.Ora Surg Oral Med Oral Patol 1972 May;33(5):841-5. [PubMed]
9. Tomov G, Iordanova M, Iordanova S. Prevalence of taurodontism among hypoand oligodontic patients. XVIII International scientific conference for young researchers. Sofia, Bulgaria, 2009; S:24756.

10. Kjaer I. Morphological characteristics of dentitions developing excessive root resorption during orthodontic treatment. Eur J Orthod 1995 Feb;17(1):2534. DOI: 10.1093/ejo/17.1.25] [PubMed]

11. Durr DP, Campos CA, Ayers CS. Clinical significance of taurodontism. J Am Dent Assoc 1980 Mar;100(3): 378-81. [PubMed]

Address for correspondence:

Dr. Miroslava Yordanova

Department of Orthodontics, Faculty of Dentistry, Medical University, Plovdiv 2, Hristo Botev Str., 4000 Plovdiv, Bulgaria

Mobile: +359898203355

E-mail:mirajord@gmail.com 